\title{
The Influence of Cognitive Psychological Bias on Library Discipline Knowledge Service
}

\section{Yu Dan}

Southwest National University Library, Chengdu, China

Email address:

yudan338@163.com

\section{To cite this article:}

Yu Dan. The Influence of Cognitive Psychological Bias on Library Discipline Knowledge Service. Science Innovation. Vol. 6, No. 1, 2018, pp. 1-3. doi: 10.11648/j.si.20180601.11

Received: December 6, 2017; Accepted: January 2, 2018; Published: March 9, 2018

\begin{abstract}
Cognitive psychology theory analysed the cause of the psychological deviation in the service and performance, and the user information demand analysis, adopt integration phase of cognitive biases and performance analysis are given, and corresponding effective countermeasures. It is of positive significance to improve the quality and efficiency of disciplinary service by taking a new perspective and looking at the entry point of disciplinary service.
\end{abstract}

Keywords: Library, Knowledge Service, Subject Librarians, Psychological Cognition, Psychological Deviation

\section{认知心理偏差对图书馆学科知识服务的影响}

余丹

西南民族大学图书馆, 成都, 中国

邮箱

yudan338@163.com

摘要: 运用认知心理学理论分析了学科服务中心理偏差的成因和表现, 并对用户信息需求、信息采纳整合阶段的认知 偏差和表现给出分析, 给出相应有效的对策。从新的视角审视学科服务的切入点, 有针对性的防范偏差和干扰因素, 对提高学科服务的质量和效率有着积极意义。

关键词: 图书馆, 知识服务, 学科馆员, 认知心理, 心理偏差

\section{1. 学科知识服务是图书馆的核心业务}

图书馆作为高校的三大门户之一, 是高等学校教学和 科研的主要信息保障体系。在当今的信息时代，信息服务 工作的效率还不能尽如人意, 使用信息服务的人还远少于 有信息需求的人。许多图书馆针对教学科研都开展了以学 科为对象的主动式信息服务: 学科馆员制。学科馆员制的 具体执行者是学科馆员, 他也是图书馆的核心力量。相对 图书馆来说, 以学科服务为主线也是图书馆业务拓展深入 开展的趋势和发展方向。
学科服务以满足用户个性化需求为目标, 并受到学科 馆员自身心理、知识及其经验的影响。实现以课程为中心、 以研究为中心、以职业为中心三种方式进行学科服务。

学科知识服务的要素与内涵, 学科服务的流程, 始于 捕获、定位用户知识信息需求, 制定工作计划和服务目标, 对相关知识信息进行采集、选择、整合、分析、创新等, 并且动态持续的为用户提供可理解的知识信息产品。这些 都建立在学科馆员的认知基础上。[1]

认知学观点的引入使得信息学研究避免了以较高机 器性能或快捷检索程序作为追求目标, 而是通过将信息用 
户的认知过程与信息行为引入信息学的研究范畴, 将信息 用户的认知行为对信息服务的影响列入了信息学的研究 重点, 以便更好地实现信息系统中的人机交互, 提高信息 服务的效率，使得信息服务更加人性化、个性化。因此服 务决策具有主观性、预见性和选择性。以助学科馆员应对 不确定性、复杂性、动态性的学科服务环境, 提高学科服 务的质量和效率。[2]

\section{2. 学科服务中认知偏差的生成因素}

图书馆业务的拓展和深化在知识服务方面, 表现为学 科馆员针对用户的个性化需要主动参与项目计划, 有目的 有计划的实施定向捕获缉拿相关学科知识信息, 完成这个 信息集纳过程, 再在自己的专业基础知识上, 记忆、存储, 通过自己的思维习惯加工, 产出新的知识体系, 再以可理 解的语言表述出, 也就完成了一个知识创新过程, 究其根 本是一个对学科知识的心理认知过程。图书馆的学科服务 也是一个学科馆员的认知活动高度和深度参与的一个过 程, 在学科服务中学科馆员还会提供动态持续的后续信息 支撑。[3]

学科服务主要从这三方面着手: 用户的信息素质教育, 逐步向深度嵌入发展; 学科支撑服务,保障基础研究的信 息需求, 着重支撑重点科研项目的发展; 学术评价服务, 从对过去绩效的评价转向为未来发展规划提供分析预测。 [4]

学科服务本身是一个知识认知高度参与的过程, 首先 学科馆员会依据用户的个性需求, 针对性的收集相关学科 知识信息, 这个信息采选过程, 所需的甄别、选择、取舍 要依据学科馆员已有的知识体系, 在此基础上再进行分析 整合创新。这其中需要学科馆员的认知, 获取用户的需求 信息及相关专业知识信息, 学习、记忆并存储, 再运用自 己的思维活动加工, 整合信息判断做出决策, 并以易于理 解的言语表述出来, 实现知识创新的一个过程。

学科服务本身是一个学科馆员认知活动高度参与的 过程, 学科馆员的认知决策在学科服务中起到关键的影响 作用, 认知偏差普遍存在人的认知活动中, 也是一个不可 避免的认知心理现象, 基于个体自身知识体系和思维加工 活动、感觉机制、个体动机和情绪情感的不同, 会使人们 对事物的认知不可避免的距离事实本身有偏离和差距。[5]

学科知识服务过程是学科馆员对用户信息需求和信 息资源环境进行匹配的过程, 其中学科馆员要运用自己的 知识结构和认知对用户的信息需求进行过滤和加工提炼, 因此对这两个因素的认知界定决定了信息服务的最后效 果。

\section{3. 对用户需求和信息资源认知的偏差表现及克 服[6]}

用户的信息需求根据用户的意识形态可以分为三类: 用户能清晰完整明确表达出来的信息需求; 用户能模糊意 识到的信息需求; 用户尚未意识到的潜在信息需求。
学科服务的开展不只是简单的信息推送和知识供给。 用户的信息需求也受到自身知识结构、学习能力、思维特 点和偏好的影响而有所不同, 随着学科服务的进展, 用户 也面临着信息的海量增长和复杂化, 用户会直接选择和自 己的信息需求完全吻合的知识信息, 通过海量信息, 过滤 䇥选, 运用自己的思维加工重构了自己的知识结构, 进而 激发出用户能明确表述出自身的信息需求, 包括潜在需求。

用户的需求能快捷完整的表述是开展学科服务在自 读者这方面的促进因素, 图书馆可以通过深度嵌入多方位 的信息素质教育来推进，提升用户获取检索信息的能力。 具体方式为专场讲座、一小时讲座、文献检索课、一对一 在线培训等。在用户一方通过提升用户信息检索获取能力, 感知能力以能快捷获知所需知识节点, 进而能完整快捷表 述所需知识信息的不足, 更快获取图书馆给于的信息回馈, 从而最终达到提高学科服务的效率和有效性。[7]

网络环境的复杂性和爆炸性增长, 容易使学科馆员出 于个体的认知偏差, 不能做到完全的将信息检索做到穷尽 性搜索以及完整的理性思考, 易采取把复杂问题简化处理

\section{4. 学科馆员对自我的认知分析}

这需要客观分析学科馆员自身的知识结构、认知风格、 思维模式以及工作习惯养成的情绪偏好, 这些决定了学科 馆员的认知策略以及对信息的处理方式。在这个对信息收 集、整理、分析、筛选和整合的过程中，馆员会不自觉选 择和自己主观意念相一致的信息, 对相反意见会做出排斥 和否决, 这样难免会主观上高估了自身能力而做出一些非 理性的判断而导致最终的学科服务结果有所偏差。正确的 认识和意识到这点, 在实际的工作中, 尽量避免主观选择, 在具体信息篎选时做到尽量客观理性的从课题需要来甄 别选择信息, 将主观情绪减小到最低程度。

\section{5. 提高学科服务的有效性, 深化和拓展图书馆 职能}

人的认知偏差是客观存在, 居于人性特点不可能完全 避免的, 我们在具体的学科服务业务中可以采取相应干预 措施最大限度来加以克服和纠偏。

激励支持策略

\section{1. 激励性刺激, 促进学科馆员的工作动力}

机构内部对职工的物质和精神双重激励, 激发员工投 入工作的最大主观能动性和最大工作热情, 激发出工作潜 能, 做出更优的学科服务决策。具体措施可以根据学科馆 员的个体情况提供相应的职业规划, 提供培训学习、深造 机会, 鼓励创新, 给予较高薪酬, 给以学科服务团队的科 研立项资助等。

\section{2. 组织馆内文化氛围加以支持}

在机构组织内积极营造一个和谐的文化环境, 为学科 馆员提供一个知识服务所必须的资源, 有一个轻松融洽的 氛围, 更便于学科馆员通过正式和非正式渠道交流和分享 
自己的学习心得 隐性知识, 实现知识和信息的共享。形 成一个平等交流的信任互动的一个关系, 尊重学科馆员的 个性工作风格。在营造的知识环境中成员交换思想心得 和经验, 鼓励开展讨论式学习团队学习学术研讨, 从而 提高馆员的自身知识能力。 [8]

\section{3. 认知改进策略}

学科馆员和用户之间存在信息不对称, 这是学科知识 服务创新的源头, 也是学科馆员在实际学科服务工作中产 生信息偏差的来源。

\subsection{1. 双向专业知识培训学习}

双向专业知识的培训学习可以促进学科馆员和用户 分享各自的知识经验, 重新建构自己的知识认知体系, 便 于彼此达成对认知的尽量接近一致, 尽大可能的趋近用户 真实的信息需求。一方面是对用户的培训, 根据信息用户 的不同需求层次开展信息资源检索技巧和专题的应用技 能、数据库使用指南、原文传递和馆际互借方法, 通过这 些方法让用户熟悉图情邻域专业术语和知识, 提高用户的 信息素养。另一方面, 用户也应该对等的向学科馆员进行 学科专业邻域的知识分享交流, 推荐本专业邻域的学术交 流和学术动态信息。[9]

\subsection{2. 反向思考（改进学科馆员的认知策略）}

学科馆员的换位思考, 从用户角度考量信息服务和服 务质量。从新的假设重新思考, 从多角度多范围选择信息 决策方案。这样更贴近用户的需要, 跟上用户信息需求的 变化, 打破自身思维的固化模式。反向思考也能在一定程 度上改进学科馆员的认知策略, 减小自身的过度自信, 从 而能有效克服学科馆员自身的认知偏差。

\subsection{3. 引入团队支持策略}

学科服务是一个知识密集型增值服务, 由知识产出过 程的各个环节的组成 人员以团队方式参与共同决策, 可 以最大限度将个人认知偏差降到最低, 有效避免因个人认 知理性不足而给学科服务带来的负面影响。学科服务是图 书馆针对用户个性化需要, 以解决用户学术专业及相关问 题为目标的知识型服务。

在实际的实践工作中, 随着经验的积累, 学科馆员要 意识到个体对知识服务中存在有认知差异, 多加强自身业 务知识学习, 克服自身的认知偏差, 尽力贴近用户的信息 需求, 准确把握用户的知识需要点, 尽最大可能满足用户 的知识需求。 $[10]$

\section{致谢}

2015年中央高校基本科研业务费专项基金项目 (项目 编号2015SZYQN182）。

\section{参考文献}

[1] 蔡金燕.美国高校图书馆学科资源建设及学科_省略_研究_ 以四所美国商学院图书馆为例 [J].新世纪图书馆, 2015 (12)

[2] (美)Sternberg, Robert J.著, 杨炳钧译. 认知心理学 [M]. 北 京:中国轻工业出版社,2006年: 479页

[3] 叶浩生. 具生认知: 认知心理学的新取向[J].心理科学进展, 2010 (5):705-709

[4] 李亚梅. 基于用户认知的学科服务可视化研究 [J]. 图书馆学 研究, 2015 (6) : 72-75,52

[5] 洪跃.学科馆员一一学科馆员发展的新模式及其实践思考 [J].图书馆学研究, 2011（3）:94-97,63

[6] 王颖, 戎文慧.学科馆员组织模式的评价与选择 [J].图书馆论 坛, 2012（5）：42-46

[7] 学科服务的多维拓展与深化_北京大学图书馆学科服务聚 焦与思考 [J].大学图书馆学报, 2012（5）:18-22

[8] 杨琳.认知心理学视阈下的情报服务研究 [D]. 黑龙江大学, 2014

[9] 郭晶.高校图书馆学科馆员能力标准与资质认证规范研究 [J].图书情报工作，2014(11):48-53

[10] 万文娟. 我国高校图书馆学科服务团队建设问题与策略分 析[J].国家图书馆学刊, 2015 (4):63-68

\section{作者简介}

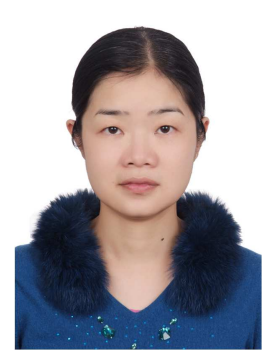

余丹, 西南民族大学图书馆学科馆员, 成都, 中国。主要研究方向: 信息行为, 学科化知识服务, 信息素养教学。 